\title{
Concomitant factor VIII inhibitor and lupus anticoagulant in an asymptomatic patient
}

\author{
Jeremy W. Jacobs ${ }^{1}$ (I) $\cdot$ Savanah D. Gisriel ${ }^{2} \cdot$ Krishna lyer $^{2} \cdot$ Henry M. Rinder ${ }^{3}$ \\ Accepted: 13 October 2021 / Published online: 25 October 2021 \\ (c) The Author(s), under exclusive licence to Springer Science+Business Media, LLC, part of Springer Nature 2021
}

\begin{abstract}
Acquired hemophilia A, caused by autoantibodies that bind to and neutralize the activity of coagulation factor VIII (FVIII), almost universally presents as a severe bleeding diathesis. Lupus anticoagulants (LAs), autoantibodies directed against phospholipids or protein-phospholipid complexes, manifest clinically with an increased risk of thrombosis. While these autoantibodies are uncommon, the distinctive clinical presentation in conjunction with the typical laboratory findings often enable straightforward identification of the underlying autoantibody. However, the presence of a concomitant acquired FVIII inhibitor and LA is exceedingly rare with fewer than 20 documented cases. All prior patients presented with life-threatening hemorrhage, thrombosis, or both, prompting comprehensive hematologic evaluation and subsequent identification of the pathologic antibodies. We describe a novel case of a patient with no signs of hemorrhage or thrombosis who was incidentally found to have both a FVIII inhibitor and LA during evaluation of a prolonged partial thromboplastin time (PTT). This finding resulted in FVIII inhibitor-directed management, including immunosuppressive therapy. The unique presentation of an incidental FVIII inhibitor and LA in an asymptomatic patient without thrombotic or bleeding complications highlights the potential challenge in elucidating the etiology of a prolonged PTT, as LAs and FVIII inhibitors both prolong the PTT, and each entity can interfere with assays designed to detect the presence of the other autoantibody. This case underscores the importance of recognizing that patients with major underlying disturbances in their hematologic physiology, but in whom clinical symptoms have yet to manifest, may potentially be overlooked until such symptoms are evident.
\end{abstract}

Keywords Autoantibodies $\cdot$ Factor VIII $\cdot$ Hemophilia A $\cdot$ Hemorrhage $\cdot$ Lupus coagulation inhibitor

\section{Highlights}

- Concomitant idiopathic coagulation factor VIII (FVIII) inhibitors and lupus anticoagulants (LAs) are rare, with fewer than 20 cases described.

- We describe the first case of an asymptomatic patient incidentally found to have both a FVIII inhibitor and LA.

Jeremy W. Jacobs

Jeremy.jacobs@yale.edu

1 Department of Laboratory Medicine, Yale School of Medicine, 330 Cedar Street, New Haven, CT 06520, USA

2 Departments of Laboratory Medicine and Pathology, Yale School of Medicine, New Haven, CT, USA

3 Department of Internal Medicine (Hematology) \& Laboratory Medicine, Yale School of Medicine, New Haven, CT, USA
- Specialized coagulation testing and interpretation are required to identify concurrent FVIII inhibitors and LAs, as these autoantibodies interfere with the typical methods used to identify the other.

- Severe coagulation abnormalities may first present with abnormal coagulation studies without apparent clinical manifestations.

- Patients with abnormal coagulation parameters should be evaluated even in the absence of symptoms.

\section{Background}

Disorders characterized by hemorrhagic or thrombotic phenotypes infrequently arise secondary to idiopathic autoantibody development. Acquired hemophilia $\mathrm{A}$ is a rare bleeding disorder caused by autoantibodies that bind to and neutralize the activity of coagulation factor VIII (FVIII). Individuals with FVIII inhibitors almost universally present with a 
bleeding diathesis, frequently to an extent that is life-threatening [1]. In contrast to the hemorrhagic features of FVIII inhibitors, lupus anticoagulants (LAs) are autoantibodies directed against phospholipids or protein-phospholipid complexes, which manifest clinically with an increased risk of thrombosis or fetal loss [2].

While these autoantibodies are uncommon, the distinct clinical presentation in conjunction with the typical laboratory findings often enable straightforward identification of the underlying autoantibody. However, the presence of a concomitant acquired FVIII inhibitor and LA is exceedingly rare with fewer than 20 documented cases (Table 1). Although the true prevalence of concurrent FVIII inhibitors and lupus anticoagulants is unknown, to date all previously described patients presented with life-threatening hemorrhage (11/16 patients), thrombosis (2/16 patients), or both (3/16 patients), prompting comprehensive hematologic evaluation and subsequent identification of the pathologic antibodies. We were unable to identify any prior report of incidental discovery of the co-occurrence of these autoantibodies. Herein, we describe the novel case of a patient with no signs or symptoms of hemorrhage or thrombosis who was incidentally found to have both a FVIII inhibitor and LA during evaluation of a prolonged partial thromboplastin time (PTT).

\section{Case presentation}

A 50-year-old woman was referred for evaluation of a prolonged PTT. She endorsed a history of cerebellar hemorrhage 10 years prior which was attributed to hypertensive emergency after she presented with a three-day history of severe headache and systolic blood pressures in the 300's. She underwent craniotomy for hematoma evacuation and blood pressure optimization, with resolution of her neurological symptoms. She denied any interim medical issues, including recurrent hemorrhage or thrombosis. At the time of cerebellar hemorrhage, the patient's coagulation studies were normal, including PTT (24.8 s; reference: 23.2-31.6 s) and prothrombin time (PT) (10.3 s; reference: $10.1-12.7 \mathrm{~s})$.

During evaluation at our institution, her PTT was prolonged (52.6 s, reference: 23.9-29.9 s), while her PT (10.4 s, reference: 9.6-12.3 s) remained normal. Upon further review, her PTT was prolonged in four studies over the previous eight months with a mean of $60.6 \mathrm{~s}$ (SD $7.2 \mathrm{~s}$; CI 53.5-67.6 s, p=0.05). She denied any history of autoimmune disorders or recent infections, including COVID-19.

Table 1 Cases of probable concomitant acquired hemophilia A and lupus anticoagulant reported in the literature, including the current patient. Modified from Ames et al. [3]

\begin{tabular}{|c|c|c|c|c|}
\hline Case \# & Authors (Year) & $\begin{array}{l}\text { Age (years) } \\
\& \operatorname{sex}(M / F)\end{array}$ & Clinical presentation & Management \\
\hline 1 & Ballard and Nyamuswa. (1993) [4] & $70 \mathrm{M}$ & Hemorrhage & FEIBA, cryoprecipitate, cyclophosphamide, steroids \\
\hline 2 & Biron et al. (1996) [5] & $92 \mathrm{~F}$ & Hemorrhage & Porcine factor VIII concentrate, steroids \\
\hline 3 & Grossmann et al. (1996) [6] & $63 \mathrm{~F}$ & Hemorrhage & $\begin{array}{l}\text { Porcine factor VIII, cyclophosphamide, immunoad- } \\
\text { sorption }\end{array}$ \\
\hline 4 & Ghirarduzzi et al. (1999) [7] & $62 \mathrm{~F}$ & Thrombosis & None (deceased) \\
\hline 5 & Saxena et al. (2000) [8] & $38 \mathrm{~F}$ & Thrombosis and hemorrhage & $\begin{array}{l}\text { CVP pulse (cyclophosphamide, vincristine, predni- } \\
\text { solone) followed by cyclophosphamide }\end{array}$ \\
\hline 6 & Saxena et al. (2000) [8] & $68 \mathrm{M}$ & Hemorrhage & Steroids and cyclophosphamide \\
\hline 7 & Saxena et al. (2000) [8] & $60 \mathrm{~F}$ & Hemorrhage & Steroids and cyclophosphamide \\
\hline 8 & Liozon et al. (2001) [9] & $64 \mathrm{~F}$ & Thrombosis & Steroids and cyclophosphamide \\
\hline 9 & Brings et al. (2002) [10] & $64 \mathrm{~F}$ & Thrombosis and hemorrhage & rFVIII, FEIBA, steroids and cyclophosphamide \\
\hline 10 & Wullen et al. (2002) [11] & $30 \mathrm{~F}$ & Hemorrhage & Steroids, IVIG \\
\hline 11 & Taher et al. (2003) [12] & $30 \mathrm{M}$ & Hemorrhage & rFVIIa, steroids \\
\hline 12 & Dreisbach et al. (2010) [13] & $59 \mathrm{M}$ & Hemorrhage & Steroids \\
\hline 13 & Spencer et al. (2011) [14] & $37 \mathrm{~F}$ & Thrombosis and hemorrhage & rFVIIa, steroids and cyclophosphamide \\
\hline 14 & Seethala et al. (2013) [15] & $60 \mathrm{~F}$ & Hemorrhage & $\begin{array}{l}\text { FEIBA, FFP, steroids, cyclophosphamide, and } \\
\text { rituximab }\end{array}$ \\
\hline 15 & Gupta et al. (2014) [16] & $69 \mathrm{~F}$ & Hemorrhage & Steroids, rFVIIa, azathioprine, IVIG, rituximab \\
\hline 16 & Belfeki et al. (2021) [17] & $77 \mathrm{M}$ & Hemorrhage & rFVIIa, steroids, cyclophosphamide \\
\hline 17 & Current patient & $50 \mathrm{~F}$ & Incidental (asymptomatic) & Prednisone, cyclophosphamide \\
\hline
\end{tabular}

$M$ male, $F$ female, FEIBA factor eight inhibitor bypassing activity, $r F V I I I$ recombinant factor VIII, $I V I G$ intravenous immunoglobulin G, $r F V I I a$ recombinant activated factor VII, FFP fresh frozen plasma 
A complete metabolic panel and complete blood count were unremarkable. Coagulation studies included a PTT mixing study that did not correct $(32.1 \mathrm{~s}$ at $0 \mathrm{~min}, 37.3 \mathrm{~s}$ following incubation at $37^{\circ} \mathrm{C}$ for $60 \mathrm{~min}$ ). LA testing demonstrated an abnormal silica clotting time (SCT) (ratio 1.28 , reference: $<1.20$ ) and an abnormal dilute Russell viper venom time (dRVVT) (ratio 1.23, reference: $<1.20$ ). Anticardiolipin IgM, IgG, and IgA antibodies and anti$\beta_{2}$-glycoprotein IgM, IgG, and IgA antibodies were not elevated. The prolonged PTT, abnormal mixing study, and abnormal SCT and dRVVT were all consistent with the presence of a LA. However, due to the abnormal PTT mixing study with near-correction at 0 min and re-prolongation at $60 \mathrm{~min}$, combined with the weak LA positivity, further testing was performed to assess for an additional coagulation factor deficiency and/or presence of a factor inhibitor.

Subsequent testing revealed markedly decreased FVIII activity (5.2\%, reference: 66-143\%) and chromogenic FVIII activity $(<11.2 \%$, reference: $50-150 \%)$. A FVIII inhibitor was detected and measured at 2.10 Bethesda units. von Willebrand factor (VWF) antigen, activity, and multimer analysis were normal. Given the findings of a FVIII inhibitor, treatment was initiated with prednisone $1 \mathrm{mg} / \mathrm{kg}$ and cyclophosphamide $2 \mathrm{mg} / \mathrm{kg}$ daily for a minimum of 6 weeks to suppress the FVIII inhibitor and mitigate bleeding risk. Table 2 depicts the trend in laboratory values for this patient.

\section{Discussion}

This unique presentation of an incidental FVIII inhibitor and LA in an asymptomatic patient without thrombotic or bleeding complications underscores the potential challenge in elucidating the etiology of a prolonged PTT, particularly in patients with multiple inhibitors. Both LAs and FVIII inhibitors prolong the PTT, and each entity can interfere with assays designed to detect the presence of the other autoantibody. A LA can interfere with PTT-based factor activity assays, resulting in a falsely decreased FVIII activity level and an erroneous diagnosis of congenital or acquired hemophilia A [18]. FVIII inhibitors, although less common than LAs, may lead to false positive results for LAs, particularly using the SCT, leading to a misdiagnosis of antiphospholipid antibody syndrome [18]. To differentiate between these two groups of autoantibodies, chromogenic FVIII activity testing and the dRVVT are valuable, as neither are affected by the presence of the other autoantibody [18]. Additionally, LAs frequently function as immediate inhibitors with no correction at $0 \mathrm{~min}$ in the PTT mixing study, while FVIII autoantibodies typically display time- and temperature-dependent inhibition [18].

This case highlights the need for detailed coagulation function analysis and comprehensive understanding of various assays' functionalities and their limitations. In practice, the indication for further laboratory testing to identify an inhibitor or factor deficiency is driven by compatible clinical findings, such as the presence of a significant bleeding disorder (e.g., coagulation factor deficiencies or inhibitors) or thrombosis (e.g., antiphospholipid antibodies). However, this patient's asymptomatic presentation necessitated specialized coagulation testing and result interpretation based on the knowledge and experience that the presence of multiple inhibitors is possible. Oftentimes, patients with laboratory abnormalities, such as a prolonged PT or PTT, are evaluated only if they endorse symptoms. Thus, patients with major underlying disturbances in their hematologic physiology, but in whom clinical symptoms have yet to manifest, may potentially be overlooked until such symptoms are evident.

These laboratory findings have important therapeutic implications for the patient presented here as well. The identification of a prolonged PTT and abnormal mixing study with partial correction, albeit not to the normal range, may lead providers to prematurely conclude that the patient had an isolated LA. Had evaluation concluded with detection of the LA, the patient would not have received appropriate management of a FVIII inhibitor, and at most would have undergone repeat LA testing $>12$ weeks later. However, the astute recognition that a weak LA did not entirely explain the partial PTT correction with mixing at $0 \mathrm{~min}$ and prolongation at $60 \mathrm{~min}$ subsequently resulted in the identification
Table 2 Patient laboratory values and therapy to date

\begin{tabular}{llll}
\hline Date & PTT $(\mathrm{s})$ & FVIII activity & Therapy \\
\hline $1 / 12$ & 69.0 & N/A & None \\
$3 / 10$ & 65.0 & N/A & None \\
$7 / 6$ & 58.0 & N/A & None \\
$8 / 26$ & 50.2 & N/A & None \\
$9 / 17$ & 52.6 & $5.2 \%$ & Prednisone $1 \mathrm{mg} / \mathrm{kg} /$ day + cyclophosphamide $2 \mathrm{mg} / \mathrm{kg} /$ day \\
$9 / 24$ & 35.8 & $25.8 \%$ & Prednisone $1 \mathrm{mg} / \mathrm{kg} /$ day + cyclophosphamide $2 \mathrm{mg} / \mathrm{kg} /$ day \\
$10 / 1$ & 28.2 & $97.4 \%$ & Prednisone $1 \mathrm{mg} / \mathrm{kg} /$ day + cyclophosphamide $2 \mathrm{mg} / \mathrm{kg} /$ day \\
\hline
\end{tabular}

PTT partial thromboplastin time, sec seconds, FVIII coagulation factor VIII 
Fig. 1 Diagnostic algorithm for detection of lupus anticoagulant and factor VIII inhibitor. PTT partial thromboplastin time, $L A$ lupus anticoagulant, $S C T$ silica clotting time, DRVVT dilute Russell viper venom time, FVIII factor VIII, AHA acquired hemophilia A, ELISA enzymelinked immunosorbent assay

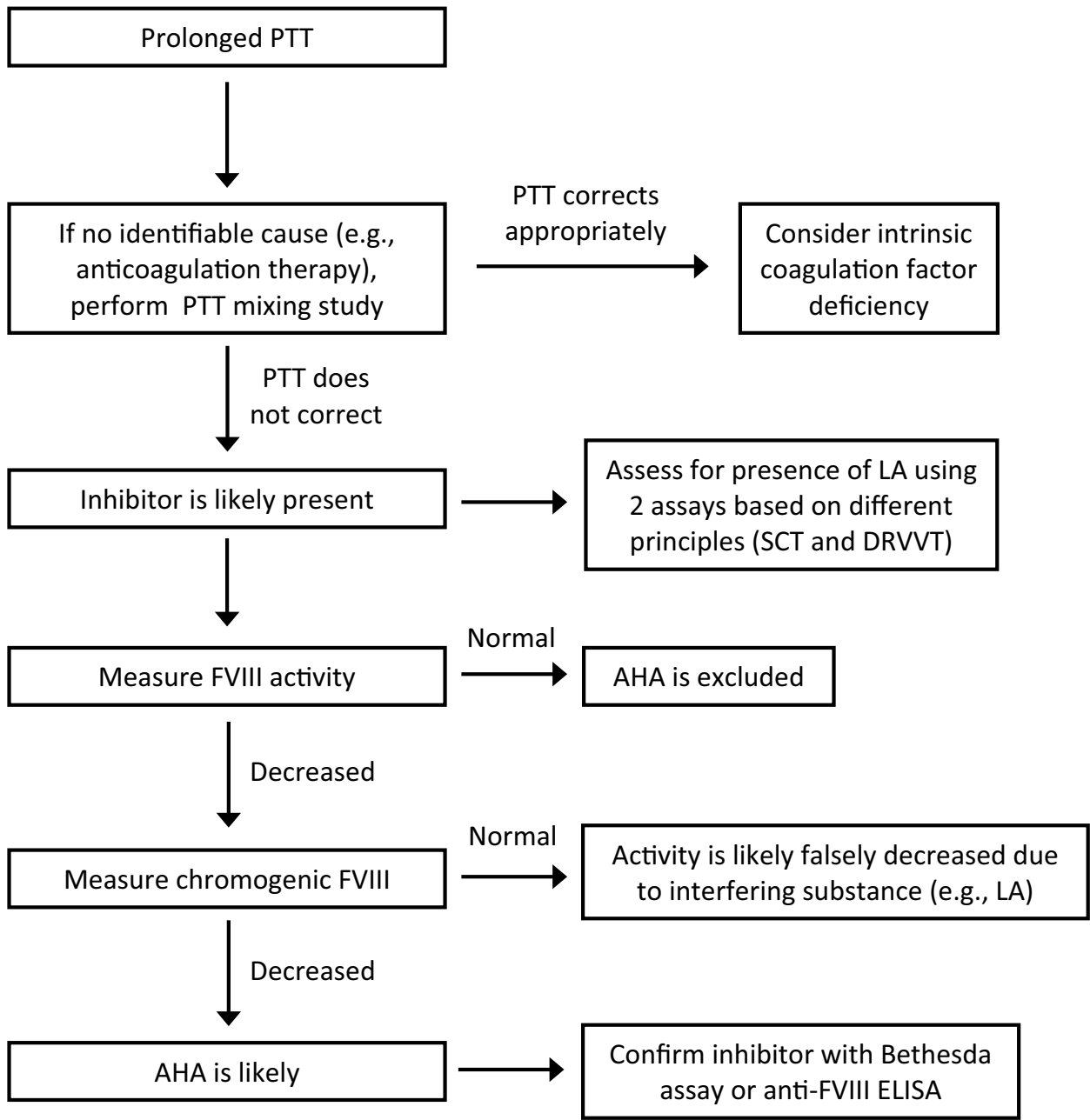

of the FVIII inhibitor. Thus, without appropriate evaluation (Fig. 1), the FVIII inhibitor might have been undetected, possibly resulting in significant morbidity or mortality. Hemorrhage in patients with acquired hemophilia A is exceptionally common ( $>90 \%$ of patients present with bleeding), and often severe (approximately $70 \%$ of patients develop severe hemorrhage) [19]. In addition to the significant risk of hemorrhage associated with FVIII inhibitors, the risk of thrombosis was also significant in our patient with a LA, as evidence demonstrates that patients with LAs have a thrombosis odds ratio 5-16 times greater than persons without LAs [20].

The detection of the FVIII inhibitor enabled the initiation of appropriate therapy. Acquired FVIII inhibitor therapy has two primary goals: to alleviate clinically relevant bleeding and to eliminate the FVIII inhibitor and mitigate the risk of hemorrhage [21]. Treatment options for achieving hemostasis in patients with FVIII inhibitors include recombinant activated factor VII and activated prothrombin complex concentrate [21]. To eliminate the autoantibody, current guidelines for immunosuppressive therapy suggest corticosteroids alone for patients with FVIII $\geq 1 \%$ and an inhibitor titer $\leq 20$ $\mathrm{BU}$ [21]. If no response is seen after 3-4 weeks, cyclophosphamide or rituximab should be added. For patients with FVIII $<1 \%$ or inhibitor titer $>20 \mathrm{BU}$, corticosteroids in combination with cyclophosphamide or rituximab is recommended [21].

Despite an inhibitor titer $<20 \mathrm{BU}$ in our patient, a more intensive regimen was warranted given her history of significant intracranial hemorrhage and overall health status. Thus, prednisone $1 \mathrm{mg} / \mathrm{kg}$ /day and cyclophosphamide $2 \mathrm{mg} / \mathrm{kg} / \mathrm{day}$ were co-administered for 6 weeks, with weekly monitoring. At the time of this report, she had received 3 weeks of immunosuppressive therapy with normalization of her PTT and FVIII activity level with no bleeding reported.

In summary, this unique case of acquired hemophilia A with a concurrent LA in a patient with a prolonged PTT without bleeding or thrombotic complications illustrates that the absence of clinical symptoms does not exclude the possibility of the co-occurrence of these autoantibodies. It also demonstrates the importance of comprehensive hematologic evaluation of patients with potential coagulation 
abnormalities, as the symptomatic manifestations may be forthcoming and life-threatening.

Authors contributions JJ designed the manuscript, analyzed and interpreted data, and wrote the manuscript. SG designed the manuscript, analyzed and interpreted data, and edited the manuscript. KI designed the manuscript, analyzed and interpreted data, and edited the manuscript. HR provided supervision, analyzed and interpreted data, edited the manuscript, and approved the final version.

Funding The authors received no funding support for this research.

Data availability N/A.

\section{Declarations}

Conflict of interest The authors declare that they have no conflict of interest relevant to the manuscript submitted to the Journal of Thrombosis and Thrombolysis.

Code availability N/A.

\section{References}

1. Kruse-Jarres R, Kempton CL, Baudo F et al (2017) Acquired hemophilia A: updated review of evidence and treatment guidance. Am J Hematol 92:695-705. https://doi.org/10.1002/ajh. 24777

2. Molhoek JE, de Groot PG, Urbanus RT (2018) The lupus anticoagulant paradox. Semin Thromb Hemost 44:445-452. https://doi. org/10.1055/s-0037-1606190

3. Ames PR, Graf M, Archer J, Scarpato N, Iannaccone L (2015) Prolonged activated partial thromboplastin time: difficulties in discriminating coexistent factor VIII inhibitor and lupus anticoagulant. Clin Appl Thromb Hemost 21:149-154. https://doi.org/ $10.1177 / 1076029614541516$

4. Ballard HS, Nyamuswa G (1993) Life-threatening haemorrhage in a patient with rheumatoid arthritis and a lupus anticoagulant coexisting with acquired autoantibodies against factor VIII. $\mathrm{Br}$ J Rheumatol 32:515-517. https://doi.org/10.1093/rheumatology/ 32.6.515

5. Biron C, Durand L, Lemkecher T (1996) Simultaneous occurrence of lupus anticoagulant, factor VIII inhibitor and localized pemphigoid. Am J Hematol 51:250-251. https://doi.org/10.1002/10968652(199603)51:3\%3c250::aid-ajh2830510302\%3e3.0.co;2-1

6. Grossmann R, Wankmüller H, Schwender S et al (1996) The diagnosis and therapy of acquired factor-VIII inhibitors in combination with lupus anticoagulants. Dtsch Med Wochenschr 121:906-910. https://doi.org/10.1055/s-2008-1043086

7. Ghirarduzzi A, Silingardi M, D'Incà M, Tincani E (1999) The antiphospholipid syndrome during chronic lymphatic leukemia. An association with anti-factor VIII antibodies. Ann Ital Med Int $14: 46-50$

8. Saxena R, Mishra DK, Kashyap R, Choudhry VP, Mahapatra M, Bhargava M (2000) Acquired haemophilia - a study of ten cases.
Haemophilia 6:78-83. https://doi.org/10.1046/j.1365-2516.2000. 00372.x

9. Liozon E, Loustaud V, Jauberteau MO et al (2001) Non-simultaneous malignant lymphoma and antiphospholipid syndrome: 4 cases. Rev Med Interne 22:360-370. https://doi.org/10.1016/ s0248-8663(01)00348-4

10. Brings HA, Waas JK, McCrae KR, Baele HR, Goldstone J (2002) Successful management of life-threatening hemorrhage in a patient with synchronous lupus anticoagulant and factor VIII inhibitor. J Vasc Surg 36:853-885. https://doi.org/10.1067/mva. 2002.127336

11. Wullen B, Mühlhöfer A, Luz H, Zoller WG (2002) Acquired hemophilia A after an early abortion. Dtsch Med Wochenschr 127:1075-1078. https://doi.org/10.1055/s-2002-30122

12. Taher A, Abiad R, Uthman I (2003) Coexistence of lupus anticoagulant and acquired haemophilia in a patient with monoclonal gammopathy of unknown significance. Lupus 12:854-856. https:// doi.org/10.1191/0961203303lu463cr

13. Dreisbach JD, Dreisbach LP, Young DE, Dreisbach PB (2010) Acquired factor VIII inhibitor and lupus anticoagulant presenting with prolonged aPTT: a case report. Grand Rounds Haematol 10:19-24

14. Spencer A, Pearce MI, Ames PR (2011) Sequential thrombosis and bleeding in a woman with a prolonged activated partial thromboplastin time. Thromb J 9:16-20. https://doi.org/10.1186/ 1477-9560-9-16

15. Seethala S, Collins NP Jr, Comerci G Jr (2013) An unusual etiology for elevation of activated partial thromboplastin time (aPTT) in SLE: acquired hemophilia and lupus anticoagulant. Case Rep Hematol 2013:521785. https://doi.org/10.1155/2013/521785

16. Gupta D, Chatterjee T, Sharma A, Ganguli P, Das S, Sharma S (2014) Rare case of acquired haemophilia and lupus anticoagulant. Indian J Hematol Blood Transfus 30(3):197-200. https://doi. org/10.1007/s12288-012-0204-5

17. Belfeki N, Hamrouni S, Strazzulla A, Diamantis S (2021) Coexistence of acquired hemophilia and antiphospholipid serology in monoclonal gammopathy patient. Int Med Case Rep J 14:261264. https://doi.org/10.2147/IMCRJ.S293931

18. Adcock DM, Favaloro EJ (2015) Pearls and pitfalls in factor inhibitor assays. Int J Lab Hematol 37(Suppl 1):52-60. https://doi.org/ 10.1111/ijlh.12352

19. Kruse-Jarres R, Kempton CL, Baudo F et al (2017) Acquired hemophilia A: updated review of evidence and treatment guidance. Am J Hematol 92(7):695-705. https://doi.org/10.1002/ajh. 24777

20. Galli M, Luciani D, Bertolini G, Barbui T (2003) Lupus anticoagulants are stronger risk factors for thrombosis than anticardiolipin antibodies in the antiphospholipid syndrome: a systematic review of the literature. Blood 101(5):1827-1832. https://doi.org/ 10.1182/blood-2002-02-0441

21. Tiede A, Collins P, Knoebl P et al (2020) International recommendations on the diagnosis and treatment of acquired hemophilia A. Haematologica 105:1791-1801. https://doi.org/10.3324/haema tol.2019.230771

Publisher's Note Springer Nature remains neutral with regard to jurisdictional claims in published maps and institutional affiliations. 